\title{
Astrometric and Spectroscopic Confirmation of a Brown Dwarf Companion to GSC 08047-00232*
}

\section{VLT/NACO deep imaging and spectroscopic observations}

\author{
G. Chauvin ${ }^{1}$, A.-M. Lagrange ${ }^{2}$, F. Lacombe ${ }^{3}$, C. Dumas ${ }^{1}$, D. Mouillet $^{4}$, B. Zuckerman ${ }^{5}$, E. Gendron ${ }^{2}$, \\ I. Song ${ }^{5}$, J.-L. Beuzit ${ }^{1}$, P. Lowrance ${ }^{6}$, and T. Fusco ${ }^{7}$ \\ ${ }^{1}$ European Southern Observatory, Casilla 19001, Santiago 19, Chile \\ ${ }^{2}$ Laboratoire d'Astrophysique, Observatoire de Grenoble, 414, Rue de la piscine, Saint-Martin d'Hères, France \\ ${ }^{3}$ Laboratoire D'Etudes Spatiales et d'Instrumentation en Astrophysique, Observatoire de Paris, Bat. 16, 5 Place \\ J. Jansen, 92195 Meudon, France \\ ${ }^{4}$ Laboratoire d'Astrophysique, Observatoire Midi-Pyrénées, Tarbes, France \\ ${ }^{5}$ Department of Physics \& Astronomy and Center for Astrobiology, Physics \& Astronomy Building, UCLA, Los \\ Angeles, CA 90095-1562, USA \\ ${ }^{6}$ SIRTF Science Center Infrared Processing and Analysis Center, MS 220-6, Pasadena, CA 91125, USA \\ ${ }^{7}$ ONERA, BP52, 29 Avenue de la Division Leclerc Châtillon Cedex, France
}

Received: / Accepted:

\begin{abstract}
We report VLT/NACO imaging observations of the stars GSC 08047-00232 and HIP 6856, probable members of the large Tucana-Horologium association. During our previous ADONIS/SHARPII deep imaging survey, a substellar candidate companion was discovered around each star. Based on VLT/NACO astrometric measurements, we find that GSC 08047-00232 and the faint candidate companion near to it share the same proper motion with a significance of $3.1 \sigma$. On the contrary, the candidate companion to HIP 6856 is probably a background object with a significance of $4.3 \sigma$. We also detect a new fainter and closer candidate companion to HIP 6856, but which is likely a background object too with a significance of $4 \sigma$. Recent VLT/NACO spectroscopic measurements of GSC 08047-00232 finally confirm the substellar nature of this young brown dwarf with a derived spectral type M9.5 \pm 1 . GSC 08047-00232 B, with an estimated mass of $25 \pm 10 \mathrm{M}_{\mathrm{Jup}}$ and an effective temperature of $2100 \pm 200 \mathrm{~K}$, is presently the third substellar companion identified among young, nearby associations.
\end{abstract}

Key words. instrumentation: adaptive optics — stars: imaging — stars: low-mass, brown dwarfs

\section{Introduction}

Since the discovery of the first bound substellar objects GD 165 B (Becklin \& Zuckerman, 1988) and Gl 229 B (Nakaiima et al., 1995), deep IR surveys have revealed the existence of numerous isolated brown dwarfs (BDs) (Moraux et al., 2003). These surveys reveal that BDs at large separations (typically $\geq 1000 \mathrm{AU}$ ) may be common as companions to stars (Gizis et al, 2001). At closer separations $(\leq 4 \mathrm{AU})$, radial velocity surveys are detecting an increasing number of planets around nearby stars and

Send offprint requests to: Gaël Chauvin e-mail: gchauvin@eso.org

* Based on observations obtained at the VLT in ESO with programs 70.C-0677 and 072.C-0644 have indicated a lack of BD companions, known as the $\mathrm{BD}$ desert (Halbwachs et al., 2000).

The intermediate separation range between 4 and $1000 \mathrm{AU}$ is just beginning to be explored as, from the ground, large ground-based telescopes coupled with adaptive optics (AO) and coronographic instruments are required. Surveying G, K and M-type nearby $(\leq 25 \mathrm{pc}$ ) stars with a typical age of $\sim 300 \mathrm{Myr}$, McCarthv \& Zuckerman (2004) recently showed that the brown dwarf desert extends from 75 to $1200 \mathrm{AU}$.

Exploring a comparable separation range with the instrument ADONIS/SHARPII at the ESO/3.6m telescope, we conducted on November 2000 and October 2001 an AO coronagraphic imaging survey of the large common Tucana-Horologium association (see Chauvin et al., 2003). This large $30 \mathrm{Myr}$ old association has been previously 
Table 1. NACO observations: instrumental set-up and observations logs. Camera S27 and S13 respectively provide 27.0 and $13.24 \mathrm{mas} /$ pixel sampling. DIT and NDIT correspond respectively to the exposure integration time and the number of exposures.

\begin{tabular}{|c|c|c|c|c|c|c|c|}
\hline Star & UT Date & $\mathrm{NDIT} \times \mathrm{DIT}$ & Filter & Camera & Mode & $\begin{array}{l}\text { Seeing } \\
(")\end{array}$ & Remarks \\
\hline \multicolumn{8}{|c|}{ Classical and Coronagraphic Imaging } \\
\hline GSC 08047-00232 AB & $23 / 11 / 2002$ & $100 \times 3 \mathrm{~s}$ & NB1.24 & $\mathrm{S} 13$ & classical & 0.82 & science \\
\hline GSC 08047-00232 AB & $23 / 11 / 2002$ & $100 \times 3 \mathrm{~s}$ & NB1.64 & $\mathrm{S} 13$ & classical & 0.99 & science \\
\hline GSC 08047-00232 AB & $23 / 11 / 2002$ & $100 \times 0.6 \mathrm{~s}$ & NB2.17 & $\mathrm{S} 27$ & classical & 0.78 & science \\
\hline GSC 08047-00232 AB & $23 / 11 / 2002$ & $100 \times 3 s$ & $\mathrm{~J}$ & $\mathrm{~S} 13$ & saturated & 0.65 & science \\
\hline GSC 08047-00232 AB & $23 / 11 / 2002$ & $100 \times 2 \mathrm{~s}$ & $\mathrm{H}$ & $\mathrm{S} 13$ & saturated & 1.05 & science \\
\hline GSC 08047-00232 AB & $23 / 11 / 2002$ & $100 \times 1 \mathrm{~s}$ & $\mathrm{Ks}$ & $\mathrm{S} 27$ & saturated & 0.69 & science \\
\hline HIP 6856 & $26 / 11 / 2002$ & $20 \times 4 s$ & $\mathrm{~J}+\mathrm{ND} 1^{a}$ & $\mathrm{~S} 13$ & classical & 0.75 & science \\
\hline HIP 6856 & $26 / 11 / 2002$ & $3 \times 60 \mathrm{~s}$ & $\mathrm{~J}$ & $\mathrm{~S} 13$ & coronagraphic & 0.72 & science \\
\hline HIP 6856 & $26 / 11 / 2002$ & $30 \times 0.34 \mathrm{~s}$ & NB2.17 & $\mathrm{S} 27$ & classical & 0.57 & science \\
\hline HIP 6856 & $26 / 11 / 2002$ & $10 \times 15 \mathrm{~s}$ & $\mathrm{Ks}$ & S27 & coronagraphic & 0.76 & science \\
\hline HD 7644 & $23 / 11 / 2002$ & $\sim 60 \mathrm{~s}$ & Each & Each & classical & $0.7-0.9$ & standard phot. \\
\hline Orion n & $21 / 11 / 2002$ & $100 \times 2 s$ & $\mathrm{H}$ & $\mathrm{S} 13$ & classical & $1.1-1.2$ & standard astro. \\
\hline HIP 6856 & $08 / 06 / 2003$ & $5 \times 4 \mathrm{~s}$ & $\mathrm{~J}+\mathrm{ND} 1^{a}$ & $\mathrm{~S} 13$ & classical & 0.85 & science \\
\hline HIP 6856 & $08 / 06 / 2003$ & $2 \times 60 s$ & $\mathrm{~J}$ & $\mathrm{~S} 13$ & coronagraphic & 0.61 & science \\
\hline HD 114842 & $29 / 05 / 2003$ & $40 \times 0.5 \mathrm{~s}$ & NB1.64 & $\mathrm{S} 13$ & classical & 0.82 & standard astro. \\
\hline HD 208372 & $30 / 05 / 2003$ & $40 \times 2 \mathrm{~s}$ & NB1.64 & $\mathrm{S} 13$ & classical & 0.39 & standard astro. \\
\hline HIP 6856 & $07 / 09 / 2003$ & $5 \times 3 \mathrm{~s}$ & $\mathrm{~J}+\mathrm{ND} 1^{a}$ & $\mathrm{~S} 13$ & classical & 0.54 & science \\
\hline HIP 6856 & $07 / 09 / 2003$ & $6 \times 60 \mathrm{~s}$ & $\mathrm{~J}$ & $\mathrm{~S} 13$ & coronagraphic & 0.56 & science \\
\hline GSC 08047-00232 AB & $07 / 09 / 2003$ & $10 \times 2 \mathrm{~s}$ & NB1.64 & $\mathrm{S} 13$ & classical & 0.6 & science \\
\hline GSC 08047-00232 AB & $07 / 09 / 2003$ & $80 \times 2.5 \mathrm{~s}$ & $\mathrm{H}$ & $\mathrm{S} 13$ & saturated & 0.75 & science \\
\hline HD 211742 & $07 / 09 / 2003$ & $5 \times 3 \mathrm{~s}$ & NB1.64 & $\mathrm{S} 13$ & classical & 0.82 & standard astro. \\
\hline GSC 08047-00232 AB & $05 / 03 / 2004$ & $4 \times 3 \mathrm{~s}$ & NB1.64 & $\mathrm{S} 13$ & classical & 0.84 & science \\
\hline GSC 08047-00232 AB & $05 / 03 / 2004$ & $4 \times 8 s$ & $\mathrm{H}$ & $\mathrm{S} 13$ & saturated & 1.12 & science \\
\hline$\theta$ Ori & $05 / 03 / 2004$ & $10 \times 12 \mathrm{~s}$ & NB2.17 & $\mathrm{S} 13$ & coronographic & $1.3-1.4$ & standard astro. \\
\hline \multicolumn{8}{|c|}{ Spectroscopy } \\
\hline GSC 08047-00232 B & $25 / 11 / 2002$ & $3 \times 600 \mathrm{~s}$ & SK-Grism & $\mathrm{S} 54$ & $\mathrm{R}=1400$ & 0.82 & science \\
\hline HIP 9022 & $25 / 11 / 2002$ & $12 \times 1 \mathrm{~s}$ & SK-Grism & $\mathrm{S} 54$ & $\mathrm{R}=1400$ & 0.68 & standard tell. \\
\hline
\end{tabular}

a ND1 is a CONICA neutral density filter with a transmission of $1.4 \%$

identified by Torres et al. (2000) and Zuckerman \& Webb (2000) with a distance estimated at $\sim 50 \mathrm{pc}$ from Earth. Based on the ADONIS/SHARPII observations of two dozen probable association members, we reported the detection of one substellar candidate companion (hereafter cc-1) to the star HIP $6856\left(\mathrm{~K} 1 \mathrm{~V}, \mathrm{~V}=9.39, d=37.1_{-1.6}^{+1.8} \mathrm{pc}\right)$ and another one to GSC 08047-00232 (K3V, $V=10.9)$. The faint object close to GSC 08047-00232 was independently detected by Neuhäuser et al. (2003) with the SHARP instrument at the ESO New Technology Telescope (NTT). Recently, Neuhäuser \& Guenther (2004) acquired $\mathrm{H}-$ and $\mathrm{K}$-band spectra of this faint object and derived a spectral type M $8 \pm 2$, but could not confirm its companionship without proper motion measurements.

Although GSC 08047-00232 is undoubtedly a young star $\left(\mathrm{W}_{\mathrm{Li}}=350 \mathrm{~m} \AA\right)$ and certainly a probable member of the Tucana-Horologium association, this star was not included among the latest volume-limited $(d \leq 60 \mathrm{pc})$ se- lection of Zuckerman \& Song (2004) in order to limit their sample size. Although no trigonometric parallaxes are known for GSC 08047-00232, a distance of 85 pc (adopted hereafter) can be derived according to the JHK photometry of the 2MASS All-Sky Catalog of Point Sources (Cutri et al., 2003). This is supported by an independent distance estimation of $87 \mathrm{pc}$ derived from a $\mathrm{V}-\mathrm{K}$ versus $\mathrm{M}_{\mathrm{K}}$ diagram (Song et al. 2004, in prep).

To study the proper motions of these faint objects, new observations were obtained with NACO at the VLT/UT4 at different epochs. NACO enabled us to detect a second closer and fainter candidate companion (hereafter cc-2) to the star HIP 6856.

We present a brief description of the observational and data reduction techniques. Then, we discuss the status of the three candidate companions based on: 1) the consistency of their photometry with the predictions obtained from evolutionary models for very low mass objects, 2) 

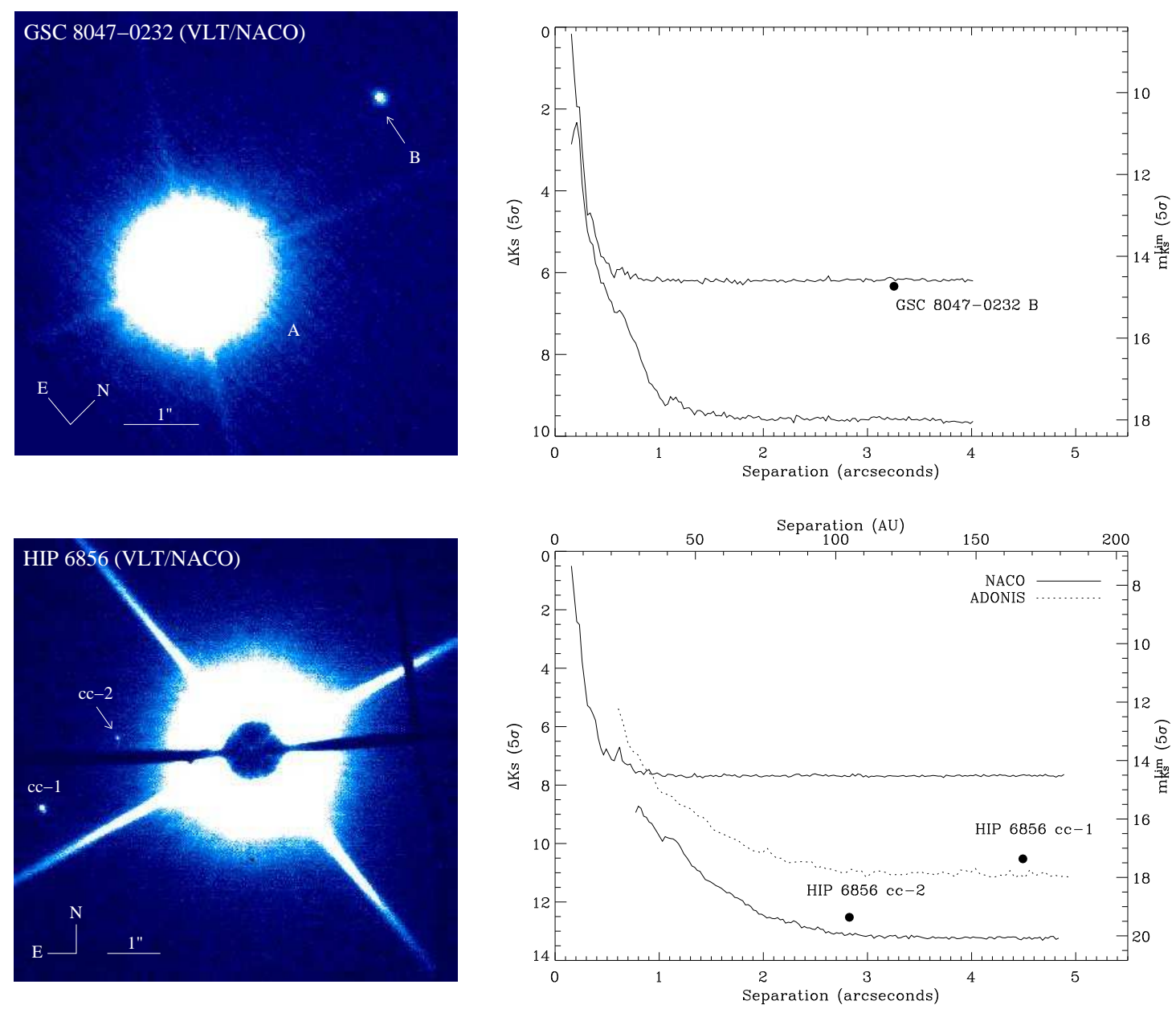

Fig. 1. Top: Left, NACO image of GSC 08047-00232 A and B observed in Ks with the S27 camera. Right, NACO (pixel to pixel) detection limits of GSC 08047-00232 obtained in classical imaging in NB2.17 $(100 \times 0.6 \mathrm{~s}$, upper solid line) and in saturated imaging Ks $(100 \times 1 \mathrm{~s}$, lower solid line $)$. Bottom: Left, Coronagraphic image of the source HIP 6856 obtained in Ks with an occulting mask of diameter 1.4" and the S27 camera. The candidate companion cc-1 $(\Delta \mathrm{Ks}=10.6)$, imaged with ADONIS/SHARPII (Chauvin et al., 2003), and the new candidate cc-2 $(\Delta \mathrm{Ks}=12.8)$, detected with VLT/NACO, are indicated. Right, NACO (pixel to pixel) detection limits of HIP 6856 obtained in classical imaging mode in NB2.17 $(5 \times 0.34 \mathrm{~s}$, upper solid line $)$ and in coronagraphy in $\mathrm{Ks}(10 \times 15 \mathrm{~s}$, lower solid line $)$. The detection limit obtained with the coronagraphic imaging mode of ADONIS/SHARPII during $15 \times 20$ s on 29 October 2001 is also reported (dotted line).

the study of their proper motion in comparison with their primary star, 3) the acquisition of a near-IR spectrum to corroborate the companionship and to confirm the substellar nature. The results of this analysis, given in sections 3,4 and 5, lead to the rejection of the two candidate companions as bound objects to HIP 6856 and the discovery of a young late-M brown dwarf companion (which we designate as B) to the star GSC 08047-00232 A.

\section{Observations and data reduction}

HIP 6856 and GSC 08047-00232 were imaged with the NAOS/CONICA (NACO) instrument of the VLT-UT4. The AO system NAOS (Rousset et al., 2002) is equipped with a tip-tilt mirror, a 185 piezo actuator deformable mirror and two wavefront sensors (Visible and IR). Attached to NAOS, CONICA (Lenzen et al., 1998) is the near infrared ( $1-5 \mu \mathrm{m}$ domain) imaging, Lyot coronagraphic, spectroscopic and polarimetric camera, equipped with a $1024 \times 1024$ pixels Aladdin InSb array.

\subsection{Classical and coronagraphic imaging}

To image the faint candidate companions to HIP 6856 , the coronagraphic mode was used with an occulting mask of diameter $1.4^{\prime \prime}$ and with the $\mathrm{J}$ and Ks filters.

In the case of the GSC 08047-00232, the narrow band filters NB1.24, NB1.64 and NB2.17 were used in classical imaging mode to avoid saturation of the primary. To image the faint candidate companion, we used the $\mathrm{J}, \mathrm{H}$ and $\mathrm{Ks}$ filters, integrating the primary above the $1 \%$ linearity level 
of the CONICA detector (i.e in saturated imaging). The relative position between the candidate companion and the primary could then be determined by taking into account the shifts between narrow band and broad band filters. The instrumental set-up and the observation logs are reported in Table 1 The detection performances achieved with VLT/NACO for these two objects are reported in Fig. [1]

Determining companionship through proper motion of the candidate companions detected with ADONIS/SHARPII was the main interest in re-imaging GSC 08047-00232 and HIP 6856. As the cc-2 to HIP 6856 was discovered thanks to NACO's detection capability, aperture photometry was used to obtain the $\mathrm{J}$ and $\mathrm{K}$ photometry of this faint object. For each epoch, the relative positions of the cc- 1 and cc- 2 to HIP 6856 were then obtained with IRAF/DAOPHOT, determining the photo-centers by a Gaussian centering. In the case of GSC 08047-00232 A and B, the deconvolution algorithm of Véran \& Rigaut (1998) was used to determine the photometry of both components but also to estimate their relative positions at each epoch. For flux calibration, the IR photometric standard HD 7644 Van der Bliek et al., 1996) was observed on November 2002 in each observing set-up used for the science objects. The photometry of the candidate companions and their primaries is reported in Table 2 The photometric measurements from the 2MASS All-Sky Catalog of Point Sources (Cutri et al., 2003) are also given and demonstrate the good fidelity of the NACO photometry in the case of GSC 08047-00232 A.

Astrometric calibrations were obtained at each epoch, by observing either the sources Orion $n$ and $\theta$ Ori from the OMC-1 complex (Luhman et al., 2000) respectively on 22 November 2002 and 5 March 2004, the astrometric references HD 114842 and HD 208372 on 29-30 May 2003, and HD 211742 on 7 September 2003. Instrumental settings and observation logs for these astrometric standards are also reported in Table 10 To obtain the best astrometric precision for our proper motion measurements, the S13 camera was used and calibrated. The orientations of true north of the S13 camera were found on 21 November 2002, 29-30 May 2003, 7 September 2003 and 5 March 2004, respectively at $-0.05^{\circ},-0.1^{\circ},+0.05^{\circ},+0.04^{\circ}$ east of the vertical within an uncertainty of $0.1^{\circ}$. The pixel scale was found stable in time with a value of $13.24 \pm 0.05$ mas. The relative positions of the candidate companions and their primaries for different epochs are reported in Table 3 The uncertainties take into account errors of the position estimation on the detector of both components as well as errors of the pixel scale and the orientation of the detector.

We did not consider here the astrometric positions determined with ADONIS/SHARPII as their uncertainty was large in comparison to the precision achieved with VLT/NACO. Neuhäuser et al. (2003) already determined the relative position of GSC 08047-00232 A and B on 4 July 2001 using speckle imaging with SHARP at the $\mathrm{ESO} / \mathrm{NTT}$ telecope. However, this measurement is not consistent (at less than $3 \sigma$ ) with the VLT/NACO relative positions that we obtained at three different epochs regarding the proper motion of GSC 08047-00232 A. Therefore, we only considered here the VLT/NACO observations to test if GSC 08047-00232 B is co-moving with GSC 08047-00232 A.

\subsection{Spectroscopy}

GSC 08047-00232 B was also observed in spectroscopy with NACO on 25 November 2002. The medium resolution $\left(\mathrm{R}_{\lambda}=1400\right)$ grism was used with the 86 mas slit, the S54 camera (54 mas/pixel) and the SK filter (1.79-2.42 $\mu \mathrm{m})$. The standard star HIP 9022 (B8/B9V) was also observed to remove the telluric lines. After substracting the sky and dividing by a flat field using eclipse (Devillar, 1997), the spectra of GSC 08047-00232 B and HIP 9022 were extracted and calibrated in wavelength with IRAF/DOSLIT. To calibrate the relative throughput of the atmosphere and the instrument, we divided the extracted spectra of GSC 08047-00232 B by the spectra of HIP 9022 and then multiplied by a blackbody to restore the shape of the continuum. GSC 08047-00232 B, separated by about $3.2^{\prime \prime}$, was not contaminated by GSC 08047 $00232 \mathrm{~A}$.

\section{Photometry and evolutionary models}

We then used the evolutionary tracks of Baraffe et al. (1998) and Chabrier et al. (2000) as a comparison to test if the photometry of the faint candidate companions is consistent with that expected for very low-mass stellar or substellar companions. The evolutionary model of Baraffe et al. (1998) is a dust-free and non-gray atmosphere model (BCAH98) appropriate to describe M dwarfs. The DUSTY and COND models of Chabrier et al. (2000) can be seen as two extreme cases, to describe respectively brown dwarfs with an atmosphere saturated

Table 2. Photometry of GSC 08047-00232 A and B (designated as GSC $08047 \mathrm{~A}$ and B below) and of the cc-1 and cc- 2 to HIP 6856

\begin{tabular}{|c|c|c|c|}
\hline Source & $\begin{array}{l}\mathrm{J} \\
(\mathrm{mag})\end{array}$ & $\begin{array}{l}\mathrm{H} \\
(\mathrm{mag})\end{array}$ & $\begin{array}{l}\text { K } \\
\text { (mag) }\end{array}$ \\
\hline $\begin{array}{l}\text { HIP } 6856^{a} \\
\text { HIP } 6856 \mathrm{cc-} 1^{b} \\
\text { HIP } 6856 \mathrm{cc-} 2^{c}\end{array}$ & $\begin{array}{l}7.41 \pm 0.02 \\
17.97 \pm 0.20 \\
19.9 \pm 0.4\end{array}$ & $6.94 \pm 0.03$ & $\begin{array}{l}6.83 \pm 0.03 \\
17.40 \pm 0.15 \\
19.6 \pm 0.2\end{array}$ \\
\hline $\begin{array}{l}\text { GSC } 08047 A^{a} \\
\text { GSC } 08047 A^{c} \\
\text { GSC } 08047 B^{c}\end{array}$ & $\begin{array}{l}9.06 \pm 0.03 \\
9.06 \pm 0.09 \\
15.90 \pm 0.13\end{array}$ & $\begin{array}{l}8.53 \pm 0.06 \\
8.54 \pm 0.05 \\
15.45 \pm 0.20\end{array}$ & $\begin{array}{l}8.41 \pm 0.03 \\
8.45 \pm 0.07 \\
14.75 \pm 0.13\end{array}$ \\
\hline $\begin{array}{l}\text { a from the } \\
\text { Cutri et al } \\
\text { brom Chauv }\end{array}$ & $\begin{array}{l}\text { IASS All-Sky } \\
\text { 2003). } \\
\text { et al. (2003). }\end{array}$ & Catalog of & Point Sources \\
\hline
\end{tabular}


Table 3. Relative positions of the cc-1 and cc-2 to HIP 6856, and of GSC 08047-00232 B to GSC 08047-00232 A .

\begin{tabular}{llllll}
\hline \hline Source & UT Date & $\begin{array}{l}\text { Sep } \\
(\mathrm{mas})\end{array}$ & $\begin{array}{l}\text { P.A. } \\
\left({ }^{\circ}\right)\end{array}$ & $\begin{array}{l}\Delta \text { Sep } \\
(\mathrm{mas})\end{array}$ & $\begin{array}{l}\Delta \text { P.A. } \\
\left({ }^{\circ}\right)\end{array}$ \\
\hline HIP 6856 cc-1 & $26 / 11 / 2002$ & $4585 \pm 14$ & $105.7 \pm 0.3$ & - & - \\
& $08 / 06 / 2003$ & $4547 \pm 14$ & $105.8 \pm 0.3$ & $-38 \pm 20$ & $+0.1 \pm 0.4$ \\
& $07 / 09 / 2003$ & $4503 \pm 13$ & $105.4 \pm 0.3$ & $-82 \pm 19$ & $-0.3 \pm 0.4$ \\
HIP 6856 cc-2 & $26 / 11 / 2002$ & $2830 \pm 13$ & $85.4 \pm 0.3$ & - & - \\
& $08 / 06 / 2003$ & $2791 \pm 15$ & $85.3 \pm 0.3$ & $-39 \pm 20$ & $-0.1 \pm 0.4$ \\
& $07 / 09 / 2003$ & $2749 \pm 14$ & $85.2 \pm 0.3$ & $-81 \pm 19$ & $-0.2 \pm 0.4$ \\
\hline GSC 08047-00232 B & $23 / 11 / 2002$ & $3274 \pm 12$ & $358.85 \pm 0.23$ & - & - \\
& $07 / 09 / 2003$ & $3266 \pm 11$ & $358.89 \pm 0.23$ & $-8 \pm 16$ & $0.04 \pm 0.31$ \\
& $05 / 03 / 2004$ & $3260 \pm 12$ & $358.82 \pm 0.22$ & $-14 \pm 15$ & $0.02 \pm 0.30$ \\
\hline
\end{tabular}

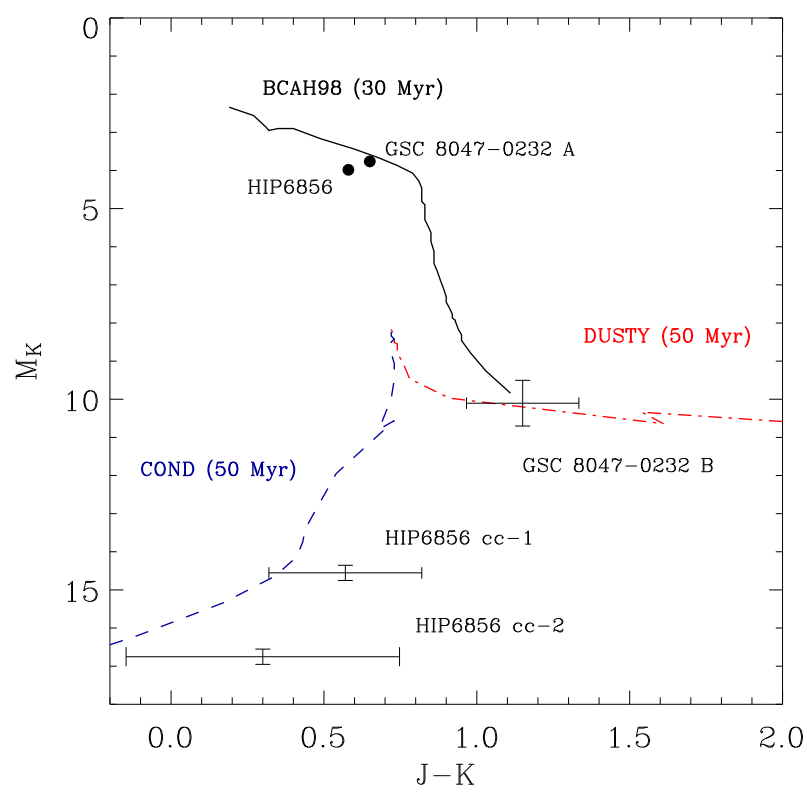

Fig. 2. Color-magnitude diagram for the near IR color $\mathrm{J}-\mathrm{K}$ from the BCAH98 model for an age of $30 \mathrm{Myr}$ and for the CBAH00 DUSTY and COND models for an age of $50 \mathrm{Myr}$ (no predictions at $30 \mathrm{Myr}$ for these two models). The data of the cc-1 and cc-2 to HIP 6856 as well as GSC 08047-00232 B are overplotted with their corresponding uncertainties. The two black points represent the two primaries HIP 6856 and GSC 08047-00232 A. As discussed previously, a distance of $85 \mathrm{pc}$ has been considered for the system GSC 08047-00232 A and B.

in dust, like late $\mathrm{M}$ and $\mathrm{L}$ dwarfs, and cool brown dwarfs or giant planets with dust condensed in their atmosphere, like T dwarfs. Based on the photometric results in Table2 the color-magnitude diagram is presented in Fig. 2

Based on the COND model predictions for an age of 10 and $50 \mathrm{Myr}$ (no predictions available between these two ages), the two candidate companions to HIP 6856 have photometry consistent with objects lighter than ten Jupiter masses. If bound, the cc-1 and cc- 2 would have respective physical separations of $180 \mathrm{AU}$ and $104 \mathrm{AU}$.
In the case of GSC 08047-00232 B, we considered a distance of of $85 \mathrm{pc}$ according to the JHKs photometry of GSC 08047-00232 A. This places GSC 08047-00232B at a projected physical separation of 278 AU. Based on the DUSTY model predictions for an age of 10 and $50 \mathrm{Myr}$, GSC 08047-00232 B has photometry consistent with a cool substellar companion with a mass of $25 \pm 15 \mathrm{M}_{\mathrm{Jup}}$ and an effective temperature of $2100 \pm 300 \mathrm{~K}$. In addition, considering the relationship between photometry and spectral type of Dahn et al. (2002), we derive a spectral type of $\mathrm{L} 0 \pm 2$ for this faint object.

\section{Proper Motions}

\subsection{Two background objects close to HIP 6856}

Based on the astrometric measurements presented in Table 3] the differences of separations for the cc- 1 to HIP 6856 between 26 November 2002 and the two successive epochs are reported in Fig. 3 3 . The expected variations (with their uncertainties) in the case of a background object are also given according to the proper motion of HIP 6856 given by the Tycho catalog (Høg et al., 2000): $\mu_{\alpha}=104.8 \pm 1.2 \mathrm{mas} / \mathrm{yr}$ and $\mu_{\delta}=-43.4 \pm 1.4 \mathrm{mas} / \mathrm{yr}$. We also considered the largest orbital variations expected in the case of a bound companion, based on Kepler's third law. The differences in terms of position angles are not considered as they are negligible in comparison to the differences in separations. Fig. 3a shows that the cc-1 is clearly not co-moving with HIP 6856 with a divergence of $4.3 \sigma$ of being a bound companion.

Similarly, we report the differences of separations for the newly discovered cc-2 to HIP 6856 (see Fig. [3b). This object is also likely a background object, with a discrepancy in separation of $4 \sigma$ of being bound.

\subsection{The co-moving system GSC 08047-00232 A and B}

According to the astrometric measurements of GSC 08047$00232 \mathrm{~A}$ and B (see Table 3), the difference in terms of separations and position angles between 23 November 2002 and the two successive epochs are reported in Fig. 3. and Fig. 3]. We used the proper motion of GSC 08047-00232 A, 

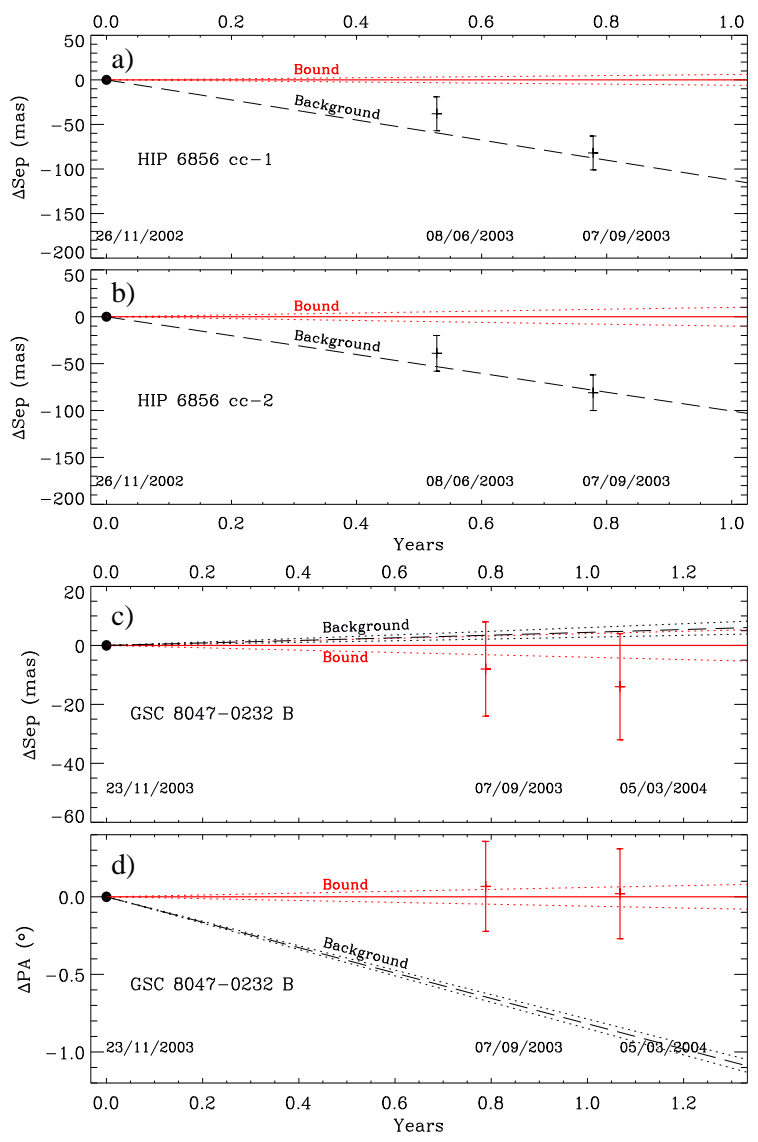

Fig. 3. a) Differences of separations, measured with NACO, for the cc-1 to HIP 6856 between $26 / 11 / 2002$ and the successive epochs 08/06/2003 and 07/09/2003. Expected evolutions with their uncertainties in case of bound (solid lines) or background (dashed lines) objects are given. b) idem but for the cc-2 to HIP 6856 . c) Differences of separations, measured with NACO, for GSC 08047-00232 B between 23/11/2002 and the two successive epochs $07 / 09 / 2003$ and $05 / 03 / 2004$. The expected evolutions with their uncertainties for bound (solid line) and background (dashed line) objects are indicated. d) idem but for the differences of position angles for GSC 08047-00232B.

$\mu_{\alpha}=46.9 \pm 1.7 \mathrm{mas} / \mathrm{yr}$ and $\mu_{\delta}=-3.1 \pm 1.7 \mathrm{mas} / \mathrm{yr}$ (Høg et al., 2000), to report also the expected variations (with their uncertainties) in the case of a background object. Contrary to the two non-true companions to HIP 6856, GSC 08047-00232 B shares common proper motion with GSC 08047-00232 A. The difference of position angles between 23 November 2002 and 5 March 2004 shows at the $3.1 \sigma$ confidence level that GSC 08047$00232 \mathrm{~B}$ is not a stationary background object.

\section{Spectral characterization of GSC 08047-00232 B}

To finally confirm the companionship of GSC08047$00232 \mathrm{~B}$ as well as its substellar nature, we recorded

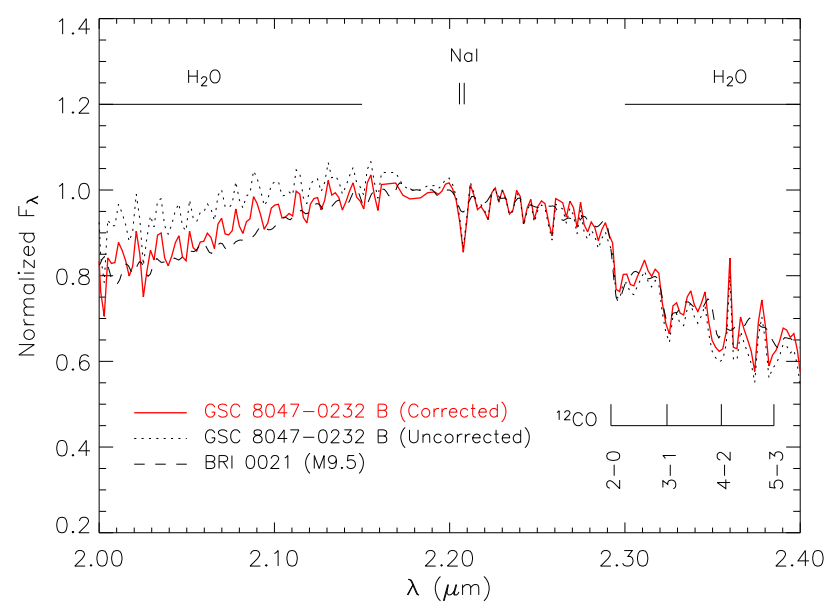

Fig. 4. SK-band spectra of GSC 08047-00232B with the medium resolution $(\mathrm{R}=1400)$ grism of CONICA, the 86 mas slit and the S54 camera (54 mas/pixel). The continuum slope of this spectrum has been increased by $14 \%$ between 2.00 and $2.48 \mu \mathrm{m}$ to best-match the template spectrum of BRI 0021 (M9.5). The spectra here have been normalized to the flux at $2.18 \mu \mathrm{m}$.

spectra on 25 November 2002 for comparison with the observations of $\mathrm{M}$, late- $\mathrm{M}$ and $\mathrm{L}$ dwarfs published by Reid et al. (2001), Geballe et al. (2002) and Leggett et al. (2001, 2000). None of these template spectra matched well the continua of the GSC 08047-00232 B spectrum which appeared to be too steep. As already evoked by Goto et al. (2002), the main plausible explanation seems to be a chromatical dependency of the flux transmitted through the narrow slit in case of a difference of centering between GSC 08047-00232 B and the standard star. This results in a decrease of the continuum slope going from short to long wavelengths, well represented by a linear function which is effectively observed in the case the GSC 08047-00232 B spectrum.

To correct this effect, we multiplied the GSC 0804700232 B spectrum by a linear function where the slope has been adjusted to match the continuum of the template spectra. The best adjustement was obtained with the template spectrum of BRI0021 (M9.5) by increasing the continuum slope of $14 \%$ between 2.00 and $2.48 \mu \mathrm{m}$

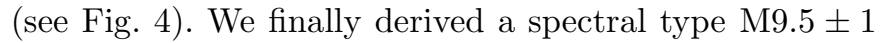
for GSC 08047-00232B based on the other template spectra. To test if such variation could be explained by the chromatical effect described previously, we simulated the flux transmitted through the 86 mas (1.6 pixels) for several NACO PSFs ${ }^{1}$. We considered different wavelengths $(2.00,2.18,2.30$ and $2.48 \mu \mathrm{m})$ and the seeing conditions of the GSC 08047-00232 B and HIP 9022 observations (see Table 11). We finally demonstrated a variation of the continuum slope of $14 \%$ between 2.00 and $2.48 \mu \mathrm{m}$ could

\begin{tabular}{|c|c|c|}
\hline 1 Simulations & obtained & with \\
\hline$N A C O$ & Preparational & Software, \\
\hline
\end{tabular}
http://www.eso.org/observing/etc/naosps-p71/doc/index.html 
be simulated with an offset of 1.2 to 1.5 pixels of the GSC 08047-00232B spectrum from the centred position behind the slit.

In addition to the continuum adjustment of GSC 08047-00232B, the presence of deep absorption lines of $\mathrm{NaI}(2.209 \mu \mathrm{m})$ and the ${ }^{12} \mathrm{CO}$ transitions $2-0$ $(2.295 \mu \mathrm{m}), \quad 3-1 \quad(2.324 \mu \mathrm{m}), \quad 4-2 \quad(2.354 \mu \mathrm{m})$ and $5-3$ $(2.385 \mu \mathrm{m})$ support the fact that GSC $08047-00232 \mathrm{~B}$ is a late-M dwarf. Using the $R_{\mathrm{CO}}$ index of Reid et al. (2001), defined as the ratio between the flux at the base of the primary band head (at $2.29 \mu \mathrm{m}$ ) and the pseudocontinuum flux at $2.27 \mu \mathrm{m}$, we find a ratio of 0.83 consistent with spectral type M8.5-M9.

Our spectral type is consistent with that of Neuhäuser \& Guenther (2004) who deduced M8 \pm 2 for GSC 08047 $00232 \mathrm{~B}$ based on $\mathrm{H}-$ and $\mathrm{K}$ - band spectra respectively obtained with VLT/ISAAC and the SOFI spectrograph at the ESO/NTT.

According to Leggett et al. (2001), the range of spectral types derived above is consistent with $\mathrm{T}_{\text {eff }}=2100 \pm$ $200 \mathrm{~K}$. This is consistent with the estimation based on the comparison between photometry and the DUSTY model predictions. Consequently, we confirm that GSC 08047$00232 \mathrm{~B}$ is a young brown dwarf companion to the star GSC 08047-00232 A, with a physical separation of $278 \mathrm{AU}$ for a distance of 85 pc. This substellar companion is presently the third one detected among the young nearby associations with TWA5 B in the TW Hydrae association, $20 \mathrm{M}_{\text {Jun }}$ at $\sim 100 \mathrm{AU}$ for $d=50 \mathrm{pc}$ (Lowrance et al., 1999; Neuhäuser et al., 2000), and HR7329 B in the $\beta$ Pictoris Group, $20-30 \mathrm{M}_{\mathrm{Jun}}$ at $\sim 200 \mathrm{AU}$ for $d=47 \mathrm{pc}$ (Lowrance et al., 2000; Guenther et al., 2001).

\section{Conclusion}

We present new VLT/NACO high contrast and high angular resolution observations of the two probable members of the Tucana-Horologium association: HIP 6856 and GSC 08047-00232. A faint candidate companion had been previously detected with ADONIS/SHARPII in the circumstellar environment of both stars (Chauvin et al., 2003). Determining companionship through proper motion and spectroscopy was the main purpose of this work.

The enhanced detection capabilities of NACO allowed us to detect a second faint object close to HIP 6856. We then checked that the photometry of these faint objects was consistent with that predicted by evolutionary model predictions for low-mass and cool objects. The two faint objects close to HIP 6856 could be consistent with fewer than ten Jupiter masses bodies and GSC 08047-00232 B with a brown dwarf companion.

Based on observations obtained at different epochs, we determined the proper motions of these faint objects and showed that the two candidate companions to HIP 6856 are likely to be background objects. On the contrary, we confirm that GSC 08047-00232 B shares common proper motion with GSC 08047-00232 A. We finally corroborate the companionship of GSC 08047-00232B based on the analysis of medium resolution spectra in the SK band. With spectral type M9.5 \pm 1 , GSC 08047-00232 B has an effective temperature of $\mathrm{T}_{\text {eff }}=2100 \pm 200 \mathrm{~K}$. According to the Chabrier et al. (2000) DUSTY evolutionary model for an age of $30 \mathrm{Myr}$, we derive a mass of $25 \pm 10 \mathrm{M}_{\mathrm{Jup}}$, confirming the substellar nature of this faint companion, the third one actually identified among young, nearby associations.

Acknowledgements. We would like to thank the staff of the ESO Paranal observatory and the NAOS/CONICA team for their help in carrying out this work. We thank also Gilles Chabrier, Isabelle Baraffe and France Allard for providing us with the latest update of their evolutionary models. We thank also Sandy Leggett, Tom Geballe and Neill Reid who kindly sent us the near-infrared template spectra of $\mathrm{M}$, late-M and $\mathrm{L}$ dwarfs for direct comparison with our measurements. Finally, we would like to thank Michael Sterzik for the different discussions about the spectroscopic analysis of $\mathrm{AO}$ observations.

\section{References}

Baraffe I., Chabrier G., Allard F. \& Hauschildt P.H., 1998, A\&A 337, 403

Becklin, E. \& Zuckerman, B. 1988, Nature, 336, 656

Chabrier, G., Baraffe, I., Allard, F. \& Hauschildt, P.H. 2000, ApJ, 542, 464

Chauvin, G., Thomson, M., Dumas, C. et al. 2003, A\&A, 406,51

Cutri, R. M., Skrutskie, M. F., van Dyk, S. et al. 2003, 2MASS All-Sky Catalog of Point Sources

Devillar N. 1997, The messenger, 87

Dahn C.C., Harris H.C., Vrba F.J. et al. 2002, AJ, 124, 1170

Elmegreen, B. G. 1999, ApJ 522, 915

Geballe, T.R., Knapp, G.R., Leggett S.K. et al. 2002, ApJ, 564, 466

Gizis, J. E., Kirkpatrick, J. D., Burgasser, A. et al. 2001, ApJ 551, 163

Goto, M., Kobayashi, N., Terada H. et al. 2002, ApJ, 567, L59

Guenther, E. W., Neuhäuser, R., Huélamo, N., Brandner, W. \& Alves, J. 2001, A\&A, 365, 514

Halbwachs, J. L., Arenou, F., Mayor, M., Udry, S. \& Queloz, D. 2000, A\&A 355, 581

Høg, E., Fabricius, C., Makarov, V.V. et al. 2000, A\&A, 355,27

Leggett, S.K., Geballe, T.R., Fan, X. et al. 2000, ApJ, 536, L35

Leggett, S.K., Allard, F., Geballe, T.R., Hauschildt, P.H. \& Schweitzer, A. 2001, ApJ, 548, 908

Lenzen, R., Hofmann, R., Bizenberger, P. \& Tusche, A., 1998, SPIE, Vol. 3354

Lowrance, P. J., McCarthy, C., Becklin, E. E. et al. 1999, ApJ, 512, L69

Lowrance, P. J., Schneider, G,, Kirkpatrick, J. et al. 2000, ApJ, 541, L390

Lucas, P.W. \& Roche, P.F. 2000, MNRAS, 314, 858 
Luhman, K., Rieke, G.H., Young, E.T. et al. 2000, ApJ, 540, 1016

McCarthy, C. \& Zuckerman, B. 2004, AJ, 127, 2871

Mizuno, H. 1980, Prog. Theo. Phys., 64, 544

Moraux, E., Bouvier, J., Stauffer, J.R. \& Cuillandre, J.-C. 2003, A\&A, 400, 891

Nakajima, T., Oppenheimer, B.R., Kulkarni, S.R. et al. 1995, Nature, 378, 463

Neuhäuser, R., Guenther, E. W., Petr, M. G. et al. 2000, A\&A, 360, 39

Neuhäuser, R., Guenther, E.W., Alves, J. et al. 2003, AN, 324,535

Neuhäuser, R. \& Guenther, E.W. 2004, A\&A, 420, 647

Pickett, B. K., Durisen, R. H., Cassen, P. \& Mejia, A. C. 2000, ApJ, 540, 95

Reid, I.N., Burgasser, A.J., Cruz, K.L., Kirkpatrick, J.D. \& Gizis, J.E. 2001, AJ, 121, 1710

Reipurth, B. \& Clarke, C. 2001, AJ, 122, 432

Rousset, G., Lacombe, F., Puget, et al., 2002, SPIE, Vol. 4007

Torres, C.A.O., Da Silva, L., Quast, G.R., de la Reza, R. \& Jilinski, E. 2000, AJ, 120, 1410

Van der Bliek, N.S., Manfroid J. \& Bouchet, P. 1996, A\&AS, 119,547

Véran, J.P. \& Rigaut, F. 1998, SPIE, 3353, 426

Zuckerman, B. \& Webb, R.A. 2000, ApJ, 535, 959

Zuckerman, B. \& Song, I. 2004, ARAA, 42, 685 